\title{
INFLUENCE OF PITTING CORROSION ON FATIGUE AND CORROSION FATIGUE OF SHIP AND OFFSHORE STRUCTURES, PART II: LOAD - PIT - CRACK INTERACTION
}

\author{
Marek Jakubowski, Assoc. Prof.
}

Gdańsk Univeristy of Technology, Poland

\begin{abstract}
In the paper has been discussed influence of stresses on general corrosion rate and corrosion pit nucleation rate and growth, whose presence has been questioned by some authors but accepted by most of them. Influence of roughness of pit walls on fatigue life of a plate suffering pit corrosion and presence of the so called "non-damaging" pits which never lead to initiation of fatigue crack, has been presented. Possibility of prediction of pit-to-crack transition moment by two different ways, i.e. considering a pit a stress concentrator or an equivalent crack, has been analyzed. Also, influence of statistical distribution of depth of corrosion pits as well as anticorrosion protection on fatigue and corrosion fatigue has been described.
\end{abstract}

Keywords: Pitting corrosion, fatigue, ship structures, offshore structures.

\section{INTRODUCTION}

Pitting corrosion constitutes the extreme form of material surface roughness due to corrosion. Melchers [1] has stated that influence of pitting corrosion on strength of well maintained structures such as ship ones is very low, but reduction of thickness due to general corrosion is much more important. However, according to common opinion, pits, if only present on material surface, constitute potential spots of fatigue crack initiation. The fact has been often, but not always, observed in research and practice [2]. At higher stress, pits in spot of corrosion crack initiation may not be present because of too short duration time. However presence of pits on material surface does not guarantee that initiation of cracks would occur in spots of the pits $[3,4]$. Thus the sequence of events: „first pit - then crack - and finally complete damage of a structure" not always takes place. There are sometimes exceptions but a great importance of the sequence of events can not be questioned.

Most publications concerning mutual pit-crack relation is focused on aircraft industry problems. Therefore they mainly concern Al-alloys with low resistance to seawater corrosion, especially of 2000 series [3, 5-7], or 7000 series [2, 8-10] of the alloys. As far as steels are concerned, majority of research papers deals with steels applied in machinery industry, i.e. medium- carbon steels after different heat treatment [11-15], or even high -carbon steels $[16,17]$ or a martensitic stainless steel [18]. Only a few papers concern weldable low-alloy, low-carbon steels applicable to ship and offshore structures [4, 19-22]. Although this paper concerns mainly ship and offshore structures, a limited amount of data on relevant materials makes that a model to be used for predicting fatigue life of such structures must be partly based on conclusions drawn from the tests on materials not applicable to ship and offshore structures. The existing models deal with aircraft materials and structures $[6,12,23,24]$. In the most complete model, initially proposed by Goswani T.K. and Hoepner D.W. (1995), and then adopted by Shi and Mahadevan [24], fatigue process runs through seven phases (Fig. 1).

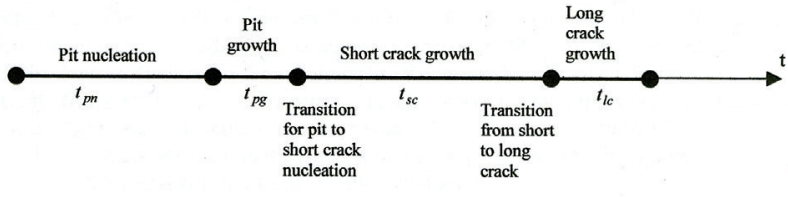

Fig. 1. Seven phases of pitting corrosion fatigue process [24]

In this approach 4 stages, each lasting a definite period, and 3 transient states: transition from pit nucleation to its growth, from pit to short crack and from short crack to long one, are considered. Phases of short crack growth and long 
crack growth are assumed to be separated from each other because of different mathematical description of short and long cracks propagation. Total pitting corrosion fatigue life of a structure, when its damaging crack is initiated in a pit, can be calculated by summing the following four phases:

$$
\mathrm{t}_{\mathrm{f}}=\mathrm{t}_{\mathrm{pn}}+\mathrm{t}_{\mathrm{pg}}+\mathrm{t}_{\mathrm{sc}}+\mathrm{t}_{\mathrm{lc}}
$$

$\mathrm{t}_{\mathrm{pn}}$ - pit nucleation duration time;

$t_{p g}$ - pit growth duration time;

$\mathrm{t}_{\mathrm{sc}} \quad$ - short crack growth duration time;

$\mathrm{t}_{\mathrm{lc}} \quad$ - long crack growth duration time;

All the above mentioned phases and transition states, or only some of them, are described deterministically $[6,12]$ or probabilistically [23,24]. Akid et al. [25], does not consider the pit- to- crack transition to be a transition state in the above presented sense but a stage which lasts a definite period. In such approach, the pit-to-crack transition time $\left(t_{p \rightarrow c}\right)$ should be included into the above presented model described by Eq.1 which takes the following form:

$$
\mathrm{t}_{\mathrm{f}}=\mathrm{t}_{\mathrm{pn}}+\mathrm{t}_{\mathrm{pg}}+\mathrm{t}_{\mathrm{sc}}+\mathrm{t}_{\mathrm{lc}}+\mathrm{t}_{\mathrm{p} \rightarrow \mathrm{c}}
$$

Phenomena, mechanism and partly the modeling of particular pitting corrosion phases has been discussed previously [26]. This paper is focused on influence of mechanical loads on pit growth kinetics as well as a criterion for transformation of pit into fatigue crack, importance of statistical distribution of pit depth with respect to corrosion fatigue life, as well as importance of anticorrosion protection in these conditions, and influence of grooving corrosion a specific kind of pitting corrosion - on fatigue of welded joints. All the phenomena discussed in this paper are controlled mainly by electrochemical action of the environment together with an additional influence of fatigue loads.

\section{INFLUENCE OF STRESSES ON GENERAL AND PITTING CORROSION}

Although this paper concerns interrelation between pitting corrosion and fatigue process, there is certain, previously described in [26], relation between general corrosion and pitting one, It is common knowledge that dynamic (monotonic or cyclic) stresses, and especially strains, increase corrosion rate of passive metalic materials such as Al-alloys and corrosion resistant steels, mainly by rupture of their passive film. However, the same was demonstrated by Evans [27] for low-carbon steel exposed to cyclic stress action in chloride solution, where the steel was covered by a very weak passive film. Corrosion rate was increasing together with increasing cyclic stresses even below fatigue limit where plastic strains are close to zero. It was probably possible due to high loading frequency, hence specimen's surface strain rate was also high. In service, ship's hull is usually exposed first of all to low-frequency loads and only from time to time to a greater change in the mean load level. Some observations indicate that every stress increase and - in smaller degree - the stress drop accelerates corrosion as a transient effect only [28]. Melchers [28] stated that at high level of strains in plate surface, rust layers are able to fall down periodically. At extremely high strains a continuous removal of rust may occur and the initially high corrosion rate may be maintained for a significant part of life of a structure. At lower strain levels rust is removed only from time to time, hence only a transient increase of corrosion rate may be expected. Melchers concluded that "the above mechanisms appears to be likely to occur only in highly strained regions, at strains well in excess of what would normally be expected in operational ships".

Kobzaruk et al. [29] tested a low-carbon steel exposed to action of natural sea environment $(1,8 \%$ salinity, $5500 \mathrm{~h}$ exposure) both without stresses and under cyclic stresses (1 cycle per day ). Corrosion of stressed coupons was faster, but more uniform with a lower number of less sharp pits than that of non-stressed ones. The fact was attributed by him to a stress-induced removal of corrosion products and marine growths, that led to a more uniform surface of the stressed specimens.

Various authors formulate different opinions about influence of stresses and strains on pitting corrosion.

Booth et al. [30] observed that the weld toe grinding, which very effectively increases fatigue life in the air for welded joints made of a high-strength low-alloy (HSLA) offshore steel, only slightly improved corrosion-fatigue life of the joints in sea water. This phenomenon resulted from pitting corrosion of the grinded surface. The mentioned slight increase of corrosionfatigue life corresponded to a short time necessary to generate pits deep and sharp enough to become crack initiation sites. They concluded that for this reason an increase of corrosion fatigue life due toe grinding is almost independent on the stresses. It means that they consider pitting corrosion rate as almost independent on stresses. For a high-carbon steel [17], under short exposure time to corrosion, growth rate of pits appears independent on applied mechanical loads. For longer exposure time values pit growth rate depends on applied loads. However, Linder and Blom [17] believe that other parameters such as flow velocity of corrosive solution and its temperature affects to a greater extent growth rate of pits. Hence, in this case the influence of cyclic stresses on pit growth rate may be neglected in engineering applications. Majority of authors, however, note a role of stresses and/or strains in pitting corrosion process.

For an austenitic steel [18] plastic strains associated with fatigue loading evidently accelerated pit nucleation in slip bands. For non-alloyed steels, influence of applied cyclic strains on pit nucleation period is not so distinct or is even negligible. Akid et al. [25] stated that the first pit appearance which was identified to be a breakdown in a moderately passive layer on steel surface, was controlled rather by electrochemical phenomena acting on metal-electrolyte interface than degree of mechanical damage. In established time periods, the mechanical damage degree was increasing along with increasing number of deformation cycles resulting from increasing load frequency (f). Akid et al. stated that the pit initiation period ti has been independent on the frequency $f$. The present author has reanalyzed the data read from an appropriate diagram published in [25] and 
stated that a weak relationship did occur and the period ti may be considered approximately proportional to $\mathrm{f}-0.105$. It means that though electrochemical control of pit nucleation dominates, mechanical loading may also play a certain role but it is not primary one. As stated in [20], the corrosion pits growing without of stresses, in an offshore structural steel, had a form close to circular on specimen surface. However along with fatigue process progress the form of the pits changed from circular to elliptical one with its main axis always perpendicular to direction of applied stresses. It proves that the stresses affected growth of pits not nucleation.

In a medium-carbon steel [13], driving force for pitting, i.e. corrosion current density inside the pit, is dependent on a stress level and its state. The pit current density, hence consequently the pit growth rate drops if the pit approaches a structural barrier of the growth, i.e. when plastic deformation range drops ahead of defect. The pit current density increases by an order of magnitude when the value of applied shear strains increases from $0.49 \%$ to $1,2 \%$, typical values for low cycle fatigue. Nakajima and Tokaji [14] stated that the average depth of pits in a similar steel under fatigue corrosion is greater than under stress-free corrosion and increases along with stress level increasing. They proposed the following relation between growth of pit depth $\left(\mathrm{a}_{\mathrm{p}}\right)$ and action of stress $(\sigma)$ :

$$
\begin{gathered}
\mathrm{a}_{\mathrm{p}}=(0.028 \sigma+0.404) \mathrm{t}^{\mathrm{B}} \\
\mathrm{B}=0.199-0.224 \\
\mathrm{a}_{\mathrm{p}}: \mu \mathrm{m} ; \mathrm{t}: \min
\end{gathered}
$$

Other tests [6] of a similar medium-carbon steel showed that the pit growth rate at $100 \mathrm{MPa}$ stress amplitude was from 5 to 10 times greater than that in load-free conditions. This order of magnitude of the pit growth rate acceleration by stresses is in agreement, for the same stress amplitude, with the value of 7 resulting from Eq. (3).

In another corrosion fatigue tests [11] on a medium-carbon steel, pits were initiated in the very early stage of the tests, i.e. approximately after 5000 load cycles (the total corrosion fatigue life was in the range from 20000 to 500000 cycles), almost independently of stress level. In non-loaded specimens the nucleation period of pits was the same but the pits ceased from growing very early. Therefore the pit growth rate increased along with stress amplitude increasing. The higher stress amplitude the greater density of pits on specimen surface. Worth reminding that in the Kobzaruk's investigations [29] influence of cyclic stresses on the density of pits on steel surface was opposite, that was probably associated with the extremely low load frequency (1 cycle per day) as well as marine growth presence.

Akid et al. [25] tested a carbon steel under fatigue loading in a synthetic sea water. They determined a ten-times increased value of pit growth rate as a result of stress range increasing. However, two threshold ranges (two plateaux) were observed where pit growth rate was almost independent of applied load range. The first of them corresponded to fatigue limit or a little above it where elastic state of surface stresses prevails. The other plateau corresponded to large stresses and the shortest fatigue lives well below 105 cycles, where "it might be considered that there is a trend towards a saturation of plasticity in the surface layers" [25]. Hence the increase of pit growth rate may be attributed mainly to the increasing range of plasticity in steel surface layers between the two plateaux. The last statement is not in compliance with some results of the tests which revealed a significant difference in pit growth rate between these observed in a load-free steel and a loaded steel below fatigue limit ) $[6,14]$.

For 2024-T3 Al-alloy [6], pit growth rate depended both on the stress amplitude $(\sigma)$ and stress frequency $(f)$. However the stress amplitude effect was distinctly dominant. The authors proposed the following formula for the growth rate of the pit $\operatorname{depth}\left(\mathrm{a}_{\mathrm{p}}\right)$ :

$$
a_{p}=A(\sigma) t^{B} f^{C}
$$

where :

C - a constant assumed equal to 0 , but its real value amounts to 0.01 ,

$\mathrm{B}=0.36$

$\mathrm{A}(\sigma)=2.34 \cdot 1.014^{\sigma}$

Wang and Akid [15] stated that the corrosion at the inclusion-matrix interface in a high-strength, medium-carbon steel, after application of cyclic stresses, run more suddenly than in the stress-free state. It leads to pit generation in this place. On the basis of nine different tests, Akid et al. [25] stated that the time to initiation of corrosion fatigue crack, i.e. the total time for nucleation and growth of a pit and its transformation into crack, was strongly decreasing along with cyclic stress increasing. Kendo et al. [31] examined a ferrite- pearlite steel in which general corrosion was non-uniform, and with corrosion pits due to non-homogenous microstructure of the steel, as well as a ferrite- bainite steel with uniform general corrosion and almost free of pits. The initiation period of corrosion fatigue crack of the first steel (with pits) was strongly shortened along with increasing level of cyclic stresses, and that of another steel (without pits) was much longer and only weakly dependent on stress level. The above mentioned results $[15,25,31]$ as well as results for Al-alloys $[8,32]$ show that pitting corrosion is accelerated by cyclic stresses.

\section{PIT-TO-CRACK TRANSITION}

When a pit reaches its critical size, often called threshold one, in its bottom a surface fatigue crack is initiated. Almost all researchers are unanimous that pit's critical size is decreasing along with increasing cyclic stress level, see e.g. $[5,6,10,11]$. At low stress amplitudes a small number of relatively large pits is generated as a result of their nucleation and growth, and fatigue cracks are always initiated in the pits, whereas at higher stress amplitudes a large number of small pits is generated in slip bands, and the test duration time is probably too short to cause pits to reach their threshold size as a result of their growth process, therefore cracks start in slip bands and only from time to time in small pits. The above described behaviour cases were the same for the steel [11] and Al-alloy [2]. 
Some authors have introduced the notion of the so called non-damaging depth of pit which never leads to fatigue crack initiation. Goto and Nisitani [11] considered pit diameter to be pit threshold size, but noted that some pits whose diameter values were greater than threshold ones, did not undergo cracks because pit depth is a more important parameter than pit diameter. Most authors mainly consider pit depth despite difficulties met in measuring pit depth in real structures. The non-damaging pit depth values are very different even for similar materials: e.g. for weldable HSLA steels the values amounted to $110 \mu \mathrm{m}$ [19] or below $40 \mu \mathrm{m}$ [21]; for Al-alloys the smallest depth of a pit in which fatigue cracks were initiated , amounted to $60 \mu \mathrm{m}$ [2], $20 \div 30 \mu \mathrm{m}$ [8] or $2 \mu \mathrm{m}$ [5], hence the non-damaging pits for Al-alloys were smaller than for steels. The above mentioned scatter of the data suggests that not only the pit depth but also another factors may affect pit-tocrack transition.

Cornet and Dolan [16] investigated an influence of salt water temperature on corrosion fatigue life of two high-carbon steels. The life was increasing along with increasing temperature of the water, because at higher temperature values pits were more of a hemispherical shape and less sharp. Therefore pit sharpness should be also taken into account. The stress concentration at a pit is determined in global scale by general pit curvature, but on the local scale by the curvature strictly in the site where crack is initiated [3]. In order to examine a role of roughness of pit walls for crack initiation in a weldable HSLA steel, many pits were chemically polished to make their bottom more smooth [19]. The life of specimens with more smooth pits was longer by about $50 \%$ than those with rough (non-polished) pits.

In global scale pit's shape is characterized by the pit aspect ratio, i.e. the ratio of the pit depth $\left(a_{p}\right)$ and pit breadth (the diameter D). The following formula has been proposed [19] for the stress concentration factor $\left(\mathrm{K}_{\mathrm{t}}\right)$ at a pit:

$$
K_{t}=1+1.25 \sqrt{\frac{2}{1+\left(\frac{D}{a_{p}}\right)^{2}}}
$$

In the formula the reciprocal of the pit aspect ratio appears. In the tests [19] the pit aspect ratio values changed in the range from $1 / 3.6$ to $1 / 2.1$ with their average value of about $1 / 3$. Hence the stress concentration factor values changed in the range from 1.85 to 2.2 with their average value equal to 1.98 . Smaller values of pit aspect ratio are often observed for steel after its very long exposure to sea environment action. For an Al-alloy the pit aspect ratio values were contained within the range from 0.5 (i.e. for hemispherical pit ) to 3,7 , and most pits were more deep than broad, hence the maximum value of stress concentration factor amounted to $\mathrm{Kt}=2,75$. The pit aspect ratio for Al-alloys shows a tendency to increasing along with increasing corrosion exposure time. It means that the real pit approximation proposed in the subject-matter literature, by using a hemispherical form of the same depth as that of real pit and the factor $K_{t}=2,25$, appears to be a conservative approach in the case of steels, but non-conservative assumption in the case ofAl-alloys. An additional support of the conclusion is the fact that walls of pits in steels are more smooth than those in $\mathrm{Al}$-alloys where there are many sharp corners resulting from a specific form of pit growing due to crystallographic tunneling [1]. Though it cannot be expected that micro-unevenness of pit walls plays a significant role in steels, but the fatigue life reduction by $30 \div 40 \%$ as a result of pit surface roughness [19] cannot be considered as negligible. The above considerations show that on the basis of the tests on Al-alloys only qualitative conclusions, but not quantitative ones, concerning pit-crack transition in steel structures, may be drawn.

The most general approach to the problem of critical depth of pit and its transition to crack was presented by Jones and Hoepner [33]. The approach was elaborated on the basis of Al-alloy tests, but it seems to be qualitatively correct also for other materials. Interaction between different factors seems to play an important role, among which the following can be counted :

- material micro-structure, its properties (especially fracture toughness) and thickness,

- $\quad$ pit depth,

- pit surface area,

- pit size and shape

- vicinity of other pits.

However for practical purposes pits on metal surface are often considered simply to act as effective stress concentrators $[3,9,19,34]$. It seems to be well-founded in the case when the pits are generated by corrosion, but later cracks in them are initiated and grown as a result of purely mechanical fatigue as in the case of aircraft fuselage structures. If a crack in a pit is initiated under corrosion fatigue conditions in a liquid or humid environment, then not only the stress concentration factor but also electrochemical activity in pit's tip should be taken into account $[18,35]$. The approach based on the stress concentration factor seems especially productive in determining fatigue life of pit-corroded material by using $\mathrm{S}-\mathrm{N}$ diagrams.

The stress concentration factors for pits, as determined with the use of Eq. (5), are independent of pit depth. However, the tests of HSLA steels [19] showed that the pit depth rather than its aspect ratio affects fatigue and corrosion fatigue properties of specimens. The life to initiation of corrosion fatigue crack and total corrosion fatigue life are decreasing when pit depth is increasing $[5,10,11]$. The fact suggests that the quantities may be mutually dependent through the stress intensity factor which constitutes the main parameter of linear-elastic fracture mechanics. Another reason for which the method is presently more and more often used, is the direct using of pit and crack dimensions in it, therefore it can be integrated with results of inspection of structures.

In this approach a corrosion pit, in the moment of its transition to crack, is simply taken as a semi-elliptical crack of the same length as the pit depth $\left(a_{\mathrm{p}}\right)[5,6,10,12,13,17,23,24]$. A question may be put how a surface defect of three-dimensional geometry and a relatively blunt tip can be approximated by a sharp twodimensional crack. However it is common knowledge that period of crack initiation from pit's tip is negligibly short 
$[5,10]$. The equating of pit growth to crack propagation is more justified since Smith and Miller [13] have shown that a crack grew from bottom of surface notch of the depth D and bottom radius $\rho$ to reach the depth 1 approximately equal to $0.13(D \rho)^{1 / 2}$, then the effective crack depth is equal to $(D+1)$. The value of 1 of the order of $1.2 \mu \mathrm{m}$ is obtained after substitution of typical values of $\mathrm{D}$ and $\rho$, equal to $20 \mu \mathrm{m}$ and $4 \mu \mathrm{m}$, respectively, where the assumed value of the radius $\rho$ accounts probably for pit bottom micro-geometry. It is rather a non-important component in crack growth calculations when pit-to-crack transition is considered[13].

Kawai and Kasai [12] applied the approach in which a pit is considered equivalent to crack, to fatigue limit determination for material with corrosion pits. They approximated Kitagawa's diagram which represents a relation between fatigue limit and crack length, by using a simple El-Haddad's relation, and determined a relation between single pit depth and fatigue limit for low-carbon steel, medium-carbon steel and a highalloy corrosion-resistant steels. The determined relations were in a satisfactory conformity with test results. However, the discussed approach in which a pit is considered as equivalent to crack, is applied much often to procedures based on linear fracture mechanics.

Range of the stress intensity factor $(\Delta K)$ value in the moment of pit-to-crack transition must be greater than, or equal to, the threshold value for propagation of fatigue or corrosion fatigue crack, $\left(\Delta \mathrm{K}_{\mathrm{th}}\right)$ :

$$
\Delta \mathrm{K} \geq \Delta \mathrm{K}_{\mathrm{th}}
$$

Kawai and Kasai proposed a relation (that can be found in [13]) for the critical depth of a pit from which a fatigue crack can subsequently grow as a function of the stress range, $\Delta \mathrm{K}_{\mathrm{th}}$, and the fatigue limits for smooth and of corroded material.

Relation (6) is valid under the assumption that initially pit grows faster than a crack in pit's root [13]. The pitting corrosion process controls pit nucleation and growth as well as a dominating part of pit-to-crack transition phase [25]. Pitting corrosion is then much faster than crack growth. Preliminary tests performed by Akid et al. [25] showed that current density in a pit dropped when crack has been initiated in it. The current density drop may be used for detecting the moment of crack initiation and determining the threshold stress intensity factor [25]. However it is not clear if the current density drop (i.e. drop of corrosion rate inside the pit) results from the crack initiation or is a condition which makes initiation of the crack inside the pit, possible. Therefore must be introduced another important condition taking into account that there is a competition between the pit growth and the crack growth. In the moment of pit-to-crack transition the pit growth rate $\left(\mathrm{da}_{\mathrm{p}} / \mathrm{dt}\right)$ must be smaller than the crack propagation rate $(\mathrm{da} / \mathrm{dt})[8,10]$ :

$$
\mathrm{da}_{\mathrm{p}} / \mathrm{dt}<\mathrm{da} / \mathrm{dt}=\mathrm{f} \cdot \mathrm{da} / \mathrm{dN} \text { at } \Delta \mathrm{K}=\Delta \mathrm{Kth}
$$

The above discussed approach is questioned by Akid et al. [25] since the pit-to crack transition is controlled not only by stress intensity but also electrochemical conditions. The linear-elastic fracture mechanics parameter $\Delta \mathrm{K}_{\mathrm{th}}$ is determined from examination of long cracks but : (i) local electrochemical conditions inside such cracks greatly differ from the conditions inside short cracks and pits, and (ii) the parameter in question concerns elastic state whereas a micro-plasticity state is associated with a pit which forms a notch.

Certainly, cracks which propagate from pits are microstructurally, physically and chemically short ones which, as it is common knowledge, propagate faster than long ones at the same value of the stress intensity factor $\Delta K$, and their propagation is possible below the $\Delta \mathrm{K}_{\mathrm{th}}$ value for long cracks. Thus $\Delta \mathrm{K}_{\mathrm{th}}$ which appears in the eq. 8 , would mean the threshold value for short cracks $[8,10]$. It seems that, in order to satisfy the condition, the crack propagation rate could be determined according to an appropriate crack growth curve for long cracks in the range of validity of Paris law but extrapolated to the threshold and near-threshold range. Such approach seems to be strongly conservative but simple. The conditions determined by Eq. (6) and/or (8) make it possible to predict an initial crack length necessary for determining the crack propagation period with the use of the methods of linear-elastic fracture mechanics. However the condition $\mathrm{da}_{\mathrm{p}} / \mathrm{dt}<\mathrm{da} / \mathrm{dt}$ is to be fulfilled during the whole crack propagation period. If it is not satisfied then pits will be formed on the crack path in the early phase of crack propagation, that was observed in some tests $[8,36]$.

Ishihara et al. [6] applied the Murakami's approach to calculate the stress intensity factor for surface cracks of an irregular shape. The authors determined the following formula for the stress intensity factor value corresponding to the moment of pit-to-crack transition:

$$
\mathrm{K}_{\mathrm{p} \rightarrow \mathrm{c}}=0.65 \sigma_{\mathrm{a}}\left\{\pi\left[(\text { area })_{\mathrm{p} \rightarrow \mathrm{c}}\right]^{1 / 2}\right\}^{1 / 2}
$$

where the areas of corrosion pits (area) $)_{p \rightarrow c}$ are those of pit traces left on the crack surfaces in the moment of their nucleation (for hemispherical pit this is the semi-circular area equal to $\pi\left(a_{p}\right)^{2} / 2$ ). The obtained $K_{p \rightarrow c}$ values, independently on their scatter, were practically independent of a level of applied stresses and approximately equal to the threshold value of the stress intensity factor, obtained from the tests of propagation rate of corrosion fatigue cracks in the rotating bending conditions.

For 7075-T6 aluminum alloy [10] pre-pitted and then fatigued in the air, the fatigue life values determined from the tests generally agreed with the predicted ones when the mean pit size was assumed to be initial crack length. The fatigue life values predicted by assuming the initial crack length to be equal to the largest pit size, were smaller than measured ones. The authors stated that much greater number of pits having dimensions more close to their mean value than to maximum one, were present. Therefore the probability of crack initiation within a mean size pit is greater. Also, Medved et al. [8] informed that in many cases the largest pits did not initiate fatigue cracks. 


\section{IMPORTANCE OF STATISTICAL PIT DEPTH DISTRIBUTION FOR CORROSION FATIGUE}

For the modeling of pitting corrosion fatigue it is necessary to know statistical distribution of depth of pits on material surface because the pit depth distribution greatly affects statistical fatigue life distribution [5]. The main effort of researchers who analyze statistical pit depth distribution, is focused on depth distribution of the largest pits. However, pits of depths closer to their mean, are more frequent and hence more hazardous from the point of view of possible initiation of fatigue cracks [10]. Therefore not only the statistical pit depth distribution but also a rigorous quantitative analysis of probability of crack initiation in pits of different depth values [10] is important for assessing fatigue damage risk for structures

\section{INTERRELATION BETWEEN CRACK INITIATION DUE TO PITTING- CORROSION FATIGUE AND ANTICORROSION PROTECTION}

In engineering practice, metal surfaces exposed to corrosive environment action are usually protected against corrosion. Cathodic protection effectively protects offshore structures against pitting corrosion $[30,37]$ which usually leads to initiation of corrosion fatigue cracks in non-protected structures. The cathodic protection will much less effectively prevent initiation of corrosion fatigue cracks if certain pits are developed on the to-be-protected surface before the protection has been applied [38].

Kumakara et al. [21] exposed specimens made of KA32 steel (with blunt notches of $\mathrm{K}_{\mathrm{t}}=2$ ) for 20 days in the air outside laboratory, which was associated with sprinkling them twice a day, i.e. every morning and evening, in order to simulate steel material storing practice in shipbuilding industry. During the period the pits up to $40 \mu \mathrm{m}$ deep were generated. Then the specimens were suitably brushed and covered with a tar epoxy resin layer. In the higher nominal stress region, cracks in the protective coating were detected earlier than crack initiation in steel. Therefore, in spite of the coating, crack initiation in steel may have a corrosion fatigue nature and start probably from pits. In the of lower nominal stresses region and longer life values, fatigue cracks were initiated without salt water interaction in previously existing pits below the coating layer, and next the coating layer was cracking as a result of increasing crack opening displacement. Salt water could penetrate through the cracks in the protective coating, therefore, in contrast to crack initiation, crack propagation in the steel preceded under corrosion fatigue conditions through the pits produced during the initial corrosion exposure. The cracks can merge plural pits initiated and developed separately.

Anticorrosive coatings are able to prevent pitting corrosion effectively, hence they can cause delay of corrosion fatigue crack initiation. Defects of a porosity type in an Al -coating thermally sprayed could result in nucleation and growth of pits on damaged surface [39]. The observation seems to be valid also for other metal and non-metal coatings, because e.g. Kumakura et al. [21\} stated that in the range of longer fatigue life values some specimens had blisters in the coating layer (tar epoxy resin), this way the coating was gradually deteriorated its quality. In the situation, corrosion developed in the steel under the protective layer.

Sometimes, to delay nucleation of pits and slow down their growth a modification of corrosion environment is implemented. For a HSLA offshore steel [20] exposed to salt water action, presence of the anodic inhibitor $\left(0.04 \mathrm{M} \mathrm{NaNO}_{3}\right)$ did not prevent pitting corrosion due to sulfide inclusions, but the pits were less numerous and showed smaller growth rates than those developed in clear salt water. Addition of an oxidizing agent to salt water $1000 \mathrm{sec}$ after application of a small anodic polarization to the pits on medium-carbon steel surface [13] resulted, after $1600 \mathrm{sec}$, in full repassivation of the pits which ceased to grow.

\section{GROOVING CORROSION VERSUS FATIGUE OF WELDED JOINTS}

Among different factors, the stress concentration factor resulting from joint's geometry is considered a factor of the greatest impact on fatigue strength. The grooving corrosion phenomenon was described in the paper [26]. High stress concentration may be expected in the case when loading direction is perpendicular to grooving corrosion line. Yuasa and Watanabe $[40,41]$ carried out tensile fatigue tests on welded butt, fillet and gusset joints made of a higher-strength steel, with grooving corrosion close to the weld edge, as well as the same tests on non-corroded joints. The initial corrosion period was equal to 3 months (Case 1) or 6 months (Case 2). Grooving corrosion rate slowed down after 3 months. Cross sections of the corroded joints are presented in Fig. 2. In the butt joints rather deep grooving corrosion (about $0.9 \mathrm{~mm}$ deep) occurred along line of the weld and close to its edge. Local corrosion grooves about 0.3 deep were observed close to the weld in the cruciform joints, but in the gusset joints almost no traces of grooving corrosion were found. The specimens were fatigued in the laboratory air atmosphere. The test results are presented in Fig. 3.

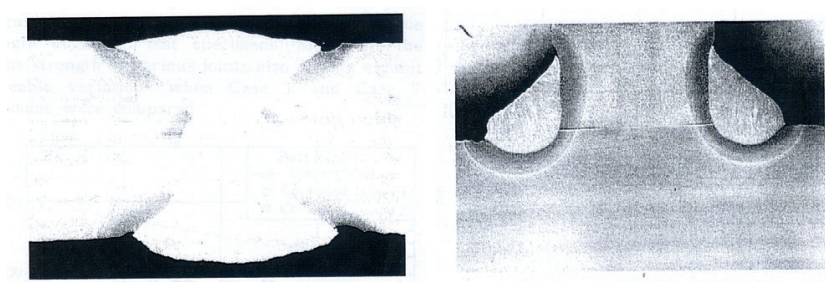

Fig.3 Cross sections of corroded specimens butt joint; cruciform joint [42].
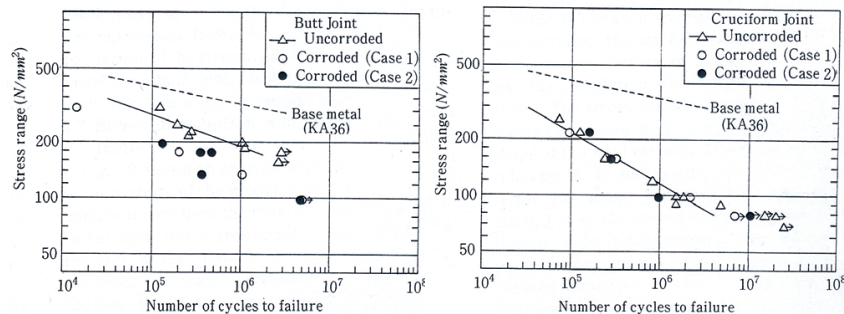

Fig.4 Results of fatigue tests in air of pre-corroded and non-corroded welded joints under tensile load of the stress ratio $R=0.1$ and at the load frequency $f=0.1 \mathrm{~Hz}[42]$. 
It has appeared that only the fatigue strength of the butt joints decreased as a result of the initial corrosion. A stress analysis showed that in this case the mean value of the stress concentration factor increased from 2.13 do 3.83 due to corrosion. The stress concentration factor values in the noncorroded cruciform joints and gusset joints (fatigue results omitted in fig. 3) were relatively high and the corrosion was not able to sharpen the notches more (in reality it even decreased the mean value of stress concentration factor from 3.91 to 3.63). Thus the fatigue strength did not almost undergo any change as a result of the pre-corrosion.

The extended pre-corrosion period did not influence fatigue properties of the joints of any type. It seems reasonable to expect that in some cases the stress concentration factor at weld toe could be even reduced due to the prolonged grooving corrosion process However in such cases a more important role may be associated rather with local reduction of plate thickness than stress concentration factor value. It depends on initial material thickness as well as loading mode.

The above given statements are correct for fillet joints with the weld leg greater than the so called critical dimension - term discussed in [42]. If the weld dimension is "subcritical" then the joint will undergo fatigue damage as a result of a fatigue crack initiated in weld root and propagating through the weld, where water has no access, hence stress concentration can not be changed under corrosion action. Then a very important role may be played by reduction of fillet weld leg, resulting from corrosion, but not by local notch sharpness at weld edge. Yuasa and Watanabe [43] modeled corrosion-induced material loss in fillet weld with the use of machining the plate and weld. Roughness of real corroded surfaces was considered unimportant because fatigue cracks were initiated in the weld root. Fatigue strength of specimens, expressed by nominal stresses, dropped markedly when weld and plate thickness has been reduced. When real, but not nominal, stress values were compared then the drop of fatigue strength of the joints, resulting from the decreased thickness of plate and weld, was much lower than in the case of nominal stresses. The above mentioned reduction of weld thickness affected also the stress concentration factor in weld root, therefore the strength of the joints with reduced thickness, expressed by real stress, showed a tendency to be somewhat smaller than that of the full - thickness joints. An inverse tendency observed in certain cases the authors attributed to the effect of releasing residual stresses as a result of reduction of weld and plate thickness.

\section{CONCLUSIONS}

1. There is the almost common opinion $[6,11,13-15,17,33]$ that growth rate of pits under stress is faster than without stress action, and that the rate increases along with applied load increasing. However in the publications the formulae for pit growth which take into account stress effect, were found only for an aluminum alloy and medium-carbon steel, both exposed to salt water for a short period under cyclic load of a relatively high frequency. A suitable formula for typical shipbuilding steels applicable in typical working conditions, especially unaerobic ones after a long exposure period, is still lacking. The opinion of Melchers [28], in which stress effect importance in these conditions is depreciated, deals mainly with general corrosion, is only qualitative one and not based on test results.

2. There are different contradictory opinions concerning applied load influence on corrosion pits:

(a) stresses do affect pitting corrosion but the stress level is a factor of secondary importance [22];

(b) plastic stresses, resulting from loading, control pit nucleation [18] and growth [30]; this could mean that stress influence in elastic range is not a significant factor and is in line with argumentation of Melchers [28] concerning general corrosion;

(c) in the case of an aluminum alloy, when load frequency increases then pit growth rate also increases (this growth is small enough to be omitted in engineering applications) [6] , but the rate evidently decreases for a HSLA steel [22];

(d) for medium-carbon steels : applied stress affects pit initation but not its growth [14], or it does not affect pit nucleation but strongly affects its growth [6].

3. Some authors $[2,5,8,19,21]$ determined the so called non-damaging pit depth, i.e. the maximum depth of pit which will never initiate a fatigue crack. The depth was greater for steel than for aluminum alloys, but for the same group of materials the depth differed significantly.

4. The critical pit depth in the moment of pit-to-crack transition decreases along with stress level increasing $[2,6,10,11]$.

5. Pit-to-crack transition seems to be a complex process dependent on many variables [34], including: (i) material micro-structure and properties, (ii) pit depth, (iii) pit size and shape, including micro-shape, (iv) pit surface area and (v) proximiti of other pits.

In a simplified, more practical approach a pit may be considered a surface crack of the same length as the pit depth. The approach makes it possible to determine: (a) fatigue limit in function of pit depth for a material with surface pits ;

(b) total fatigue life of a material with surface pits, by using the methods of linear-elastic fracture mechanics.

6. The following working conditions should be checked in order to determine the critical pit depth:

(a) $\Delta \mathrm{K} \geq \Delta \mathrm{K}_{\text {th }}$

(b) $\mathrm{da}_{\mathrm{p}} / \mathrm{dt}<\mathrm{da} / \mathrm{dt}=\mathrm{f} \cdot \mathrm{dd} / \mathrm{dN}$ at $\Delta \mathrm{K}=\Delta \mathrm{K}_{\text {th }}$

(c) to the relation (a) the threshold value of the stress intensity factor $\left(\Delta \mathrm{K}_{\mathrm{th}}\right)$ for short cracks should be inserted, or $\Delta \mathrm{K}_{\mathrm{th}}=0$ should be assumed; in the latter case the condition (b) will be decisive of pit depth critical value.

7. Statistical pit depth distribution was described by some authors, but the descriptions were focused on the distribution of maximum values only, whereas it seeems [10] that the mean value of pit depths controls the mean value of fatigue life of specimens. 
8. Cathodic protection effectively prevents pitting corrosion, but already existing pits developed during storing steel material in open air or during a break between protection periods can greatly reduce fatigue life of a protected structure.

9. Fatigue damages affect pitting corrosion: (a) degradation of protective coatings may lead, in corrosion fatigue conditions, to occurrence of blisters in the coatings and formation of pits under a metal or non-metal coating, (b) fatigue crack initiation in a pit results in a transient drop of corrosion current inside the pit.

10. Tar epoxy resin coating greatly extends corrosion fatigue life of steel [21], but does not guarantee full protection for it because :

(a) at higher nominal stress amplitude values (i.e. shorter life values) the coating layer frequently suffered cracks before a crack has been initiated in the steel; it means that the crack initiation and propagation processes in steel were running in corrosion fatigue conditions;

(b) at lower nominal stress amplitude values cracks were initiated in the pits developed under the protective coating during the pre-exposure of steel material in open air and after sprinkling it twice a day; then the coating was fractured as a result of increasing crack opening displacement and water penetrated into the cracks which continued to propagate but now in corrosion fatigue conditions.

11. In the case of the joints with a lower stress concentration, i.e. butt joints, grooving corrosion occurring in heat affected zone close to weld edge resulted in a marked reduction of fatigue strength. In the case of the joints with a higher stress concentration, i.e. cruciform and gusset joints, the notch at weld toe become even blunted by the grooves, hence the corrosion did not decrease fatigue strength of the joints. It seems that the corrosion grooves, similarly to pits, may be considered straight -line- front cracks or through-thickness cracks.

\section{ACKNOWLEDGEMENTS}

This work has been performed in the scope of the project RISPECT "Risk-Based

Expert System for Through-Life Ship Structural Inspection and Maintenance and New-Build Ship Structural Design" which has been financed by the UE under the contract SCP7-GA-2008-218499

\section{REFERENCES}

1. Melchers R.E.: Pitting corrosion of mild steel in marine immersion environment - Part 1: Maximum pit depth. Corrosion, 2004, Vol.60, No 9, pp.824-836.

2. Chlistovsky, Hefferman P.J., DuQuesnay D.L., Corrosion fatigue behavior of 7075-T651 aluminum alloy subjected to periodic overloads, International Journal of Fatigue, 2007, vol.29, pp.1941-1949
3. van der Walde K., Hillberry B.M., Initiation and shape development of corrosion nucleated fatigue cracks, International Journal of Fatigue, 2007, Vol.29, pp.1269-1281

4. Lu B.T., Luo J.L.: Crack initiation and early propagation of X70 steel in near-neutral pH groundwater, Corrosion, 2006, No 8, Vol.62, pp. 723-731

5. Dolley E.J., Lee B., Wei R.P.: The effect of pitting corrosion on fatigue life. Fatigue \& Fracture of Engineering Materials \& Structures, 2000, Vol.23, pp.555-560

6. Ishihara S., Saka S., Nan Z.Y., Goshima T., Sunada S., Prediction of corrosion fatigue lives of aluminium alloy on the basis of corrosion pit growth law, Fatigue \& Fracture of Engineering Materials \& Structures, 2006, Vol.29, pp.472480

7. Pidapatri R.M., Patel R.R.: Correlation between corrosion pits and stresses in Al alloys. Materials Letters 2008, Vol.62, pp.4497-4499.

8. Medved J.J., Breton M., Irving P.E.: Corrosion pit size distribution and fatigue lives - a study of the EIFS technique for fatigue design in the presence of corrosion. International Journal of Fatigue 2004, vol.26, pp.71-80.

9. Pao P.S., Gill S.J., Feng C.R.: On fatigue crack initiation from corrosion pits in 7075-T7351 aluminum alloy, Scripta Mater., 2000, Vol. 43, 391-396

10. Sankaran K.K., Perez R., Jata K.V.: Effect of pitting corrosion on the fatigue behavior of aluminum alloy 7075-T6: Modeling and experimental studies, Materials Science and Engineering, 2001, Vol. A297, pp223-229 10

11. Goto M., Nisitani H.: Crack initiation and propagation behavior of a heat-treated carbon steel in corrosion fatigue. Fatigue \& Fracture of Engineering Materials \& Structures, 1992, Vol.15, pp.353-36311

12. Kawai S., Kasai K.: Considerations of allowable stress of corrosion fatigue (focused on the influence of pitting), Fatigue \& Fracture of Engineering Materials \& Structures, 1985, Vol.8, pp.115-127 12

13. Miller K.J., Akid R.: The application of microstructural Fracture Mechanics to various metal surface states. Proc. Royal Society A, 1996, Vol. 452, 1411-1432 13

14. Nakajima M., Tokaji K.: Fatigue life distribution and growth of corrosion pits an a medium carbon steel in $3 \% \mathrm{NaCl}$ Solution. Fatigue \& Fracture of Engineering Materials \& Structures, 1995, Vol.18, pp.345-351 14

15. Wang Y., Akid R. Role of nonmetallic inclusions in fatigue, pitting and corrosion fatigue. Corrosion, 1996, Vol. 52, p.92-104 15 
16. Cornet i., Golan S.: Influence of temperature on corrosion fatigue. Corrosion, 1959, No 5, p.262 16

17. Linder J., Blom R.: Development of a method for corrosion fatigue life prediction of structurally loaded bearing steel. Corrosion, 2001, Vol. 57, No.5, pp.404-412 17

18. Qian Y.R., Cahoon J.R.: Crack initiation mechanisms for corrosion fatigue of austenitic stainless steel. Corrosion, 1997, Vol.53, No.2, pp.129-135 18

19. Ahin S.-H. Lawrence Jr. F.V., Metzger M.M.: Corrosion fatigue of an HSLA steel. Fatigue \& Fracture of Engineering Materials \& Structures, 1992, Vol.15, pp.625-642 19

20. Boukerrou A., Cottis R.A.: Crack initiation in the corrosion fatigue of structural steels in salt solutions. Corrosion Science, 1993, Vol.35, pp.577-585 20

21. Kumakura Y., Takanashi M., Fuji A., Kitagawa M., Ojima M., Kobayashi Y.: Fatigue strength of coated steel plate in seawater. Proc. Ninth Int. Offshore and Polar Engineering Conference, Brest, France, May 30 - June 4, 1999, Vol.4, pp. 108-113. 21

22. Maximovich, Kobzaruk: Initiation and propagation of low-cycle fatigue cracks in 15HN5DMF steel in seawater. Physical Chemical Mechanics of Materials,1985, Vol.20, No 5, pp.16-20 (in Russian) 22

23. Shi P., Mahadevan S.: Probabilistic corrosion fatigue life prediction. 8th ASCE Specialty Conference Probabilistic Mechanics and Structural Reliability. 200023

24. Zhang R., Mahadevan S.: Reliability based reassessment of corrosion fatigue life. Structural Safety, 2001, Vol.23, pp.77-91 24

25. Akid R., Dmytrakh I.M., Gonzales-Sanchez J.: Fatigue damage accumulation: the role of corrosion on the early stages of crack development. Corrosion Engineering, Science and Technology, 2006, Vol.41, No.4, pp.328-335. 25

26. Jakubowski M., Influence of pitting corrosion on fatigue and corrosion fatigue of ship structures. Part 1:Mechanisms and modeling of pitting corrosion of ship structures. Polish Maritime Research,

27. Evans U.R., Tohopandui Simnad M., The mechanism of corrosion fatigue of mild steel. Proceedings of the Royal Society, Series A,1947, vol.188, pp.372-392. 27

28. Melchers R.E., Development of new applied models for steel corrosion in marine applications including shipping. SAOS, 2008, Vol.3, No2, pp.135-144. 28

29. Kobzaruk K.A.V., Marichev V.A.: Corrosion and corrosion fatigue resistance of steels in real marine and in laboratory. Physical Chemical Mechanics of Materials,1981, Vol.16, No 2, pp.15-21 (in Russian) 29
30. Booth G.S.: Constant amplitude corrosion fatigue strength of welded joints. Fatigue in Offshore Structural Steels (Proc. of a Conference London, 24-25 Feb., 1981), Paper No 2, pp.5-16 30

31. Konda N., Suzuki S., Tada N., Kho Y., Kazushige A., Watanabe E., Yamamoto $M$ and Yaima H.: Effect of microstructure on fatigue properties of steel in seawater - developement of steels for high resistance to fatigue in ships, Part 2. J Soc. Naval Architects of Japan, 2001, Vol.191, pp.229-237. 32

32. Rajasankar J., Iyer N.Y., Gopinath S., Probabilistic modeling of fatigue crack initiation from pits and pit clusters in aluminum alloys, Corrosion Engineering, Science and Technology, 2007, Vol.42, No.3, pp.260-265 33

33. Jones K, Hoeppner D.W., Prior corrosion and fatigue of 2024-T3 aluminum alloy, Corrosion Science, 2006, Vol.48, pp.3109-3122 34

34. Grimes D., i in.: Corrosion fatigue strength of welded $\mathrm{K}$-joints and HSLA-cast steel hybrid K-nodes at component-similar scale. Steels in Marine Structures, Amsterdam 1987, pp.465-478.

35. Ebara R.: Corrosion fatigue phenomena learned from failure analysis, Engineering Failure Analysis, 2006, Vol.13, pp.516-525 36

36. Sonsino C.M., Lipp K., Lachman E.: Corrosion fatigue of welded high-strength cast and structural steel joints under constant and variable amplitude loading. Proc. Fifth Int. Offshore and Polar Conference, The Hague, June 11-16, 1995, pp.53-58. 37

37. Jootsen M.W., Salama N.N.: Corrosion fatigue of aluminum sprayed, high strength steel immersed in seawater. Material Performance, 1984, Vol.23, No 7, pp.22-26 38

38. Booth G.S.: Techniques for improving the corrosion fatigue strength for plate welded joints. Steel in Marine Structures, Amsterdam, 1987, pp.747-757 39

39. Connolly B.J., Meng Q., Moran A.L., McCaw R.L., Mechanical and pre-corroded fatigue properties of coated aluminum aircraft skin system as function of various thermal spray processes, Corrosion Engineering, Science and Technology, 2004, Vol.39, No.2, pp.137-142 40

40. Yuasa M, Watanabe T.: Fatigue strength of corroded weld joints. J. Society of the Naval Architects of Japan, 1994, Vol.176, pp.481-490 (in Japanese) 41

41. Yuasa M, Watanabe T.: Fatigue strength of corroded weld joints. ClassNK Technical Bulletin, 1996, Vol.14, pp.51-61 (in English) 42 
42. Gurney T.R.: Fatigue of welded structures. (monograph) Cambridge University Press, 196843

43. Yuasa M., Watanabe T.: The influence of corrosion wastage on the fatigue strength of fillet welded joints. NK. Tech. Bulletin, 1998, pp.21-31. 44
CONTACT WITH AUTHOR

Marek Jakubowski

Gdańsk University of Technology Ocean Engineering and Ship Technology Narutowicza 11/12 Str. 80-233 Gdansk POLAND 